\title{
Nódulos radiculares en coníferas chilenas
}

\author{
Root nodules in Chilean conifers \\ C.D.O. 164.3 - $174.7-181.36$
}

RENATO GREZ Z.

Instituto de Silvicultura, Facultad de Ciencias Forestales, Universidad Austral de Chile, Casilla 567, Valdivia, Chile.

\section{SUMMARY}

Root nodulation in Chilean conifers is described, particularly in Araucariaceae and Podocarpaceae. The nodules are spherical and brownish yellow in Podocarpaceae -varying in diameter among species- while in Araucariaceae they are like elongated reddish spheres.

\section{RESUMEN}

Se describe la existencia de nódulos radiculares en coníferas chilenas, particularmente en Araucariaceae y Podocarpaceae. Los nódulos presentan forma esférica y color amarillo pardo en Podocaipaceae -variando en diámetro según especie- y forma esferoidal elongada, con color rojizo en Araucariaceae.

\section{INTRODUCCION}

La nodulación en sistemas radiculares de coniferas, en particular de algunas Araucariaceae y Podocarpaceae, ha sido descrito desde hace aproximadamente cien años (Bevege et al. 1978).

En Chile, si bien existen varias especies representantes de coniferas, éstas no han sido objeto de estudios referentes a sus sistemas radiculares en cuanto a nodulación (Donoso', Ramírez², 1988, comunicación personal).

Considerando la importancia que representan ciertos tipos de nodulación, no sólo como un elemento descriptivo de la morfología radicular, sino que desde la perspectiva de su capacidad de fijación de nitrógeno atmosférico, se ha considerado importante estudiar la presencia de estos cuerpos en las especies chilenas Araucaria araucana (Mol). C. Koch (familia Araucariaceae), Saxegothaea conspicua Lindl. (Mañío de hojas cortas), Podocarpus nubigena Lindl. (Mañío de hojas punzantes), Podocarpus saligna D. Don (Mañío de hojas largas) y Prumnopitys andina (Poepp. ex. Endl.) de Laub. (Lleuque). Además, y con el propósito de corroborar la existencia de nodulación en otra especie de araucariáceas, se incluyó Araucaria angustifolia (Bert.) O. Ktze. (Pino de Paraná), cuyo lugar de origen es Río Negro, Paraná, Brasil.

\section{MATERIAL Y METODOS}

Se recolectaron raíces de las especies referidas anteriormente, procedentes de los lugares señalados en el cuadro 1. Estas corresponden a árboles de diferente edad y seleccionadas al azar. Las raíces fueron lavadas con agua y sometidas a ultrasonido para eliminar restos de suelo adherido a ellas; luego fueron secadas al aire y observadas bajo lupa estereoscópica, para su caracterización morfológica y toma de fotografías. Los colores descritos fueron referidos a tablas Munsell.

\section{RESULTADOS Y DISCUSION}

Todas las especies estudiadas presentaron formación de nódulos, independientemente de su lugar de ubicación o procedencia. Esto se pudo constatar no sólo en las raíces recolectadas sino que además en todos los ejemplares observados en terreno.

\footnotetext{
${ }^{1 .}$ C. DONOSO, Instit. Silvicultura, Fac. Ciencias Forestales, Univ. Austral de Chile.

2. C. RAMIREZ, Inst. Botánica, Fac. Ciencias, Univ. Austral de Chile.
} 
Tal hecho, que se había detectado con anterioridad en Podocarpus nubigena, fue descrito por el autor de este trabajo con motivo de un reconocimento edáfico-vegetacional efectuado en el sector litoral insular de Aysén (Peñaloza, R., R. Grez, C. Donoso y L. Cerda, 1982). Del mismo modo, la nodulación pudo ser confirmada en otros reconocimientos de suelos afectados en la Novena y
Décima Región de Chile, indistintamente de la condición edafoclimática imperante.

En cuanto a la morfología, los nódulos de las Podocarpaceae son similares entre sí, de igual forma, como sucede entre los nódulos de las Araucariaceae. Sin embargo, entre ambos casos se presentan diferencias importantes (Fig. 1).

En las Podocarpaceae predomina la forma

\section{Cuadro 1}

Procedencia de raíces de coniferas chilenas consideradas en el estudio.

Origin of Chilean conifer roots studied

\begin{tabular}{|c|c|c|}
\hline $\begin{array}{l}\text { Conifera } \\
\text { (Número de muestras) }\end{array}$ & Lugar & Suelo o sustrato \\
\hline \multicolumn{3}{|l|}{ Araucariaceae } \\
\hline Araucaria araucana(2) & $\begin{array}{l}\text { Valdivia, Vivero } \\
\text { Experimental, Fac. } \\
\text { Ciencias Forestales, } \\
\text { Univ. Austral Chile }\end{array}$ & $\begin{array}{l}\text { Suelo derivado de cenizas } \\
\text { volcánicas, Trumao. }\end{array}$ \\
\hline \multicolumn{3}{|l|}{ Podocarpaceae } \\
\hline $\begin{array}{l}\text { Saxegothaea } \\
\text { conspicua Lindl.(4) }\end{array}$ & $\begin{array}{l}\text { Valdivia, } \\
\text { Cordillera } \\
\text { de la costa }\end{array}$ & $\begin{array}{l}\text { Suelo derivado de rocas } \\
\text { metamórficas micaesquísti- } \\
\text { cas, y suelo de arenas sedi- } \\
\text { mentarias. }\end{array}$ \\
\hline $\begin{array}{l}\text { Podocarpus } \\
\text { nubigena Lindl.(4) }\end{array}$ & $\begin{array}{l}\text { Valdivia } \\
\text { Cordillera } \\
\text { de la costa y } \\
\text { valle central }\end{array}$ & $\begin{array}{l}\text { Suelo derivado de rocas } \\
\text { metamórficas micaesquísti- } \\
\text { cas y suelo derivado de ceni- } \\
\text { zas volcánicas, } \\
\text { Trumao. } \\
\text { Tronco de Alerce. }\end{array}$ \\
\hline $\begin{array}{l}\text { Podocarpus }(S) \\
\text { saligna D. Don }\end{array}$ & $\begin{array}{l}\text { Valdivia, Vivero } \\
\text { Experimental y } \\
\text { Arboreto, Facultad } \\
\text { Ciencias Forestales, } \\
\text { Univ. Austral Chile }\end{array}$ & $\begin{array}{l}\text { Suelo derivado de cenizas } \\
\text { volcánicas y suelo mezcla de } \\
\text { cenizas volcánicas con rocas } \\
\text { metamórficas micaes- } \\
\text { quísticas. }\end{array}$ \\
\hline $\begin{array}{l}\text { Prumnopitys }(4) \\
\text { andina (Poepp. ex } \\
\text { Endl.) de Laub. }\end{array}$ & Valdivia, Arboretum & $\begin{array}{l}\text { Suelo derivado de cenizas } \\
\text { volcánicas sobre rocas } \\
\text { metamórficas. }\end{array}$ \\
\hline $\begin{array}{l}\text { Araucariaceae } \\
\text { Araucaria(8) } \\
\text { angustifolia }\end{array}$ & Valdivia, Arboretum & $\begin{array}{l}\text { Suelo derivado de cenizas } \\
\text { volcánicas sobre rocas } \\
\text { metamórficas. }\end{array}$ \\
\hline
\end{tabular}




\section{NODULOS RADICULARES EN CONIFERAS CHILENAS}

esférica, color amarillo pardo $10 \mathrm{YR}$ 6/6, con un diámetro aproximado entre 0.2 y $1.0 \mathrm{~mm}$, encontrándose adheridos alrededor de las raíces finas y medias (Fig. 1/ A, B, C y D).

En Podocarpus nubigena los nódulos alcanzan comparativamente mayores tamaños y cantidad, y su distribución es regular y diametralmente simétrica y opuesta al eje radicular (Figs. 1 y 2). En condiciones de sustrato menos nutritivo, por ejemplo, en suelo ácido, derivado de rocas

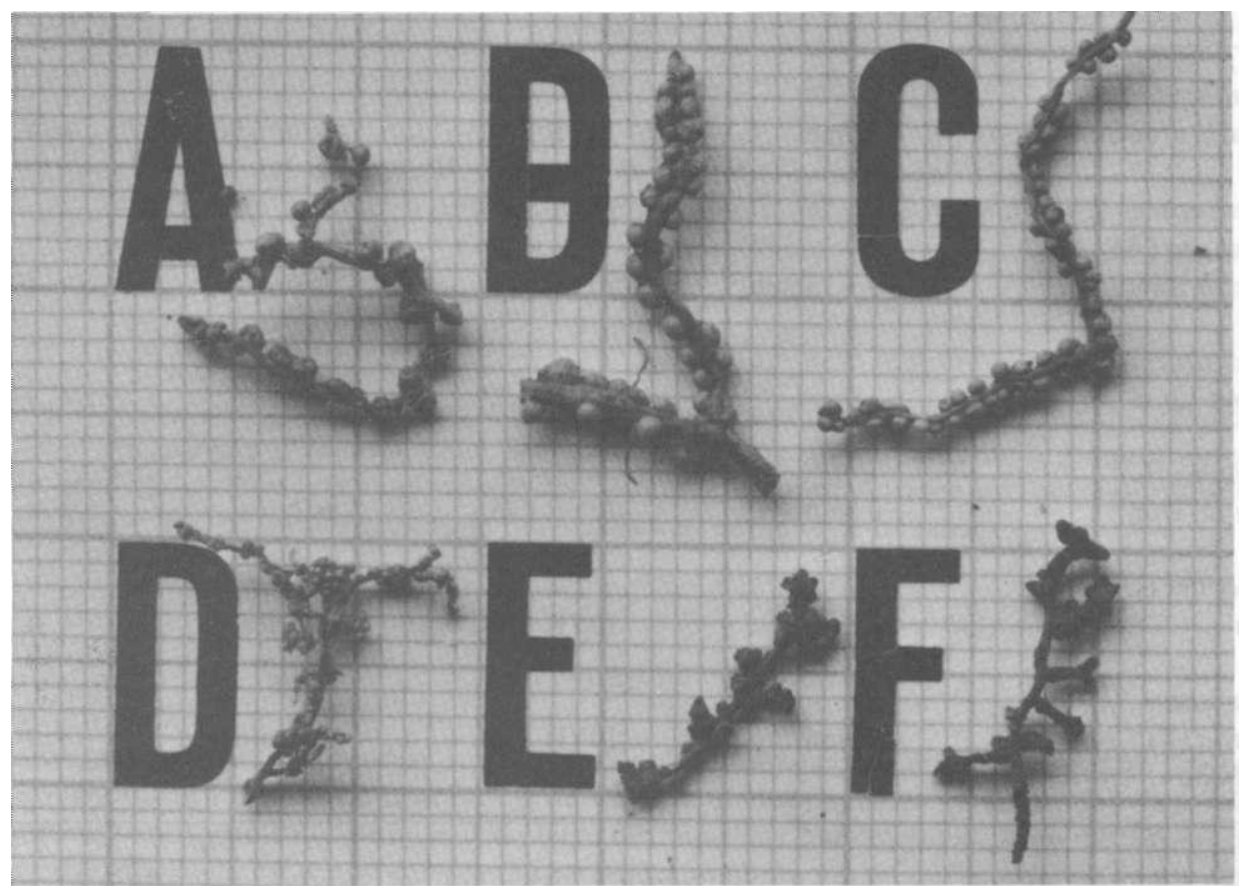

Fig. 1: Nodulación radicular en Podocarpaceae y Araucariaceae.

Root nodulation in Podocarpaceae and Araucariaceae.

$\mathrm{A}=$ Saxegothaea conspicua, $\mathrm{B}=$ Podocarpus nubigena, $\mathrm{C}=$ Podocarpus saligna, $\mathrm{D}=$ Prumnopifys andina, $\mathrm{E}=$ Araucaria araucana, y F= Araucaria angustifolia.

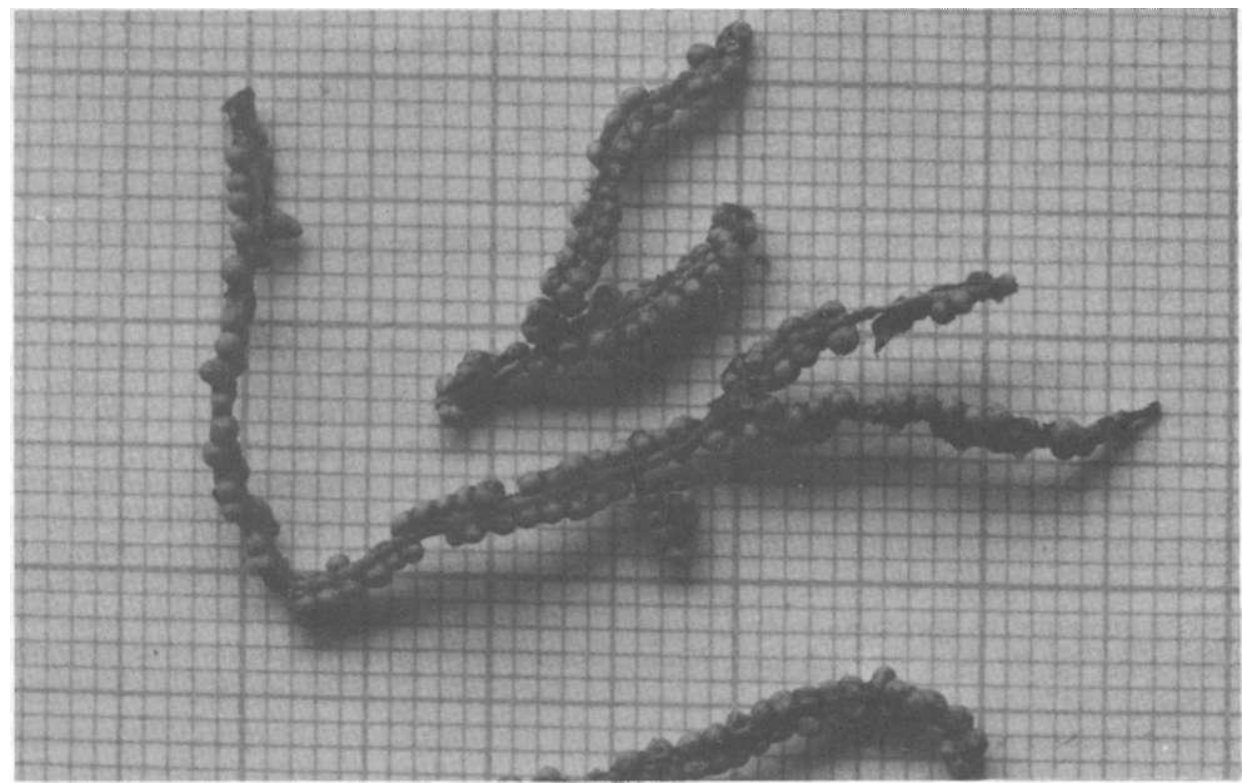

Fig.2: Nodulación radicular en Podocarpus nubigena.

Root nodulation in Podocarpus nubigena. 


\section{CUADRO 2}

Características morfológicas principales de nódulos radiculares en coniferas chilenas

Main morphological characteristics of root nodules in Chilean conifers

\begin{tabular}{|c|c|c|c|c|}
\hline Especie & Tamaño & Color & Forma & $\begin{array}{l}\text { Cantidad } \\
\text { Relativa }\end{array}$ \\
\hline Araucariaceae & & & & \\
\hline Araucaria araucana (E) & $\sim 0.5 \mathrm{~mm}$ & $10 Y R 3 / 4$ & esferoidal & ++ \\
\hline Podocarpaceae & & & & \\
\hline $\begin{array}{l}\text { Saxegothaea (A) } \\
\text { conspicua Lindl. }\end{array}$ & $\sim 0.5-1.0 \mathrm{~mm}$ & 10YR 6/6 & esférica & +++ \\
\hline $\begin{array}{l}\text { Podocarpus (B) } \\
\text { nubigena Lindl. }\end{array}$ & $-1.0 \mathrm{~mm}$ & 10YR 6/6 & esférica & ++++ \\
\hline $\begin{array}{l}\text { Podocarpus (C) } \\
\text { saligna D. Don }\end{array}$ & $\sim 0.5 \mathrm{~mm}$ & 10YR 6/6 & esférica & ++++ \\
\hline $\begin{array}{l}\text { Prumnopitys (D) } \\
\text { andina }\end{array}$ & $\sim 0.2 \mathrm{~mm}$ & 10YR 6/6 & esférica & +++ \\
\hline $\begin{array}{l}\text { Araucariaceae } \\
\text { Araucaria }(\mathrm{F}) \\
\text { angustifolia }\end{array}$ & $\sim 0.5 \mathrm{~mm}$ & $10 Y R 3 / 4$ & esferoidal & ++ \\
\hline
\end{tabular}

metamórficas o en restos de un tronco de Alerce, la nodulación es más abundante. En las especies restantes la nodulación no presenta un ordenamiento tan marcado y el tamaño de éstos es menor.

Los nódulos emergen de la rizodermis que cubre las raíces finas. Posteriormente esta epidermis tiende a desaparecer cuando las raíces adquieren una consistencia leñosa por el crecimiento secundario, permaneciendo los nódulos adheridos directamente y en forma individual a las raíces.

Observaciones realizadas en nódulos de Podocarpus nubigena permiten señalar que poseen una cavidad central, la cual se encuentra llena de fluido líquido o gaseoso, según sea el régimen hídrico existente en el medio en que se desarrollan.

A diferencia de lo descrito anteriormente, en las Araucariaceae los nodulos se caracterizan por una forma esferiodal elongada, color rojo oscuro $10 \mathrm{YR} 3 / 4$, con un diámetro aproximado de $0.5 \mathrm{~mm}$ y adheridos a las raíces finas sin ordenamiento definido (Fig. 1). Al extraer el nodulo de la raíz, queda adherido a ésta un tejido parenquimático que se asemeja a una prolongación radicular, cosa que no se observó en las Podocarpaceae.

Un resumen de las características antes descritas se presenta en el cuadro 2, individualizando para cada especie la identificación referida en la Fig. 1.

\section{BIBLIOGRAFIA}

BEVEGE, D.I.; RICHARDS, B.N. Y MOORE, A.W. 1978. Nitrogen Fixation Associated with Conifers. Division of Soils Divisional Report N ${ }^{\circ} 26$, CSIRO, Australia.

MUNSELL SOIL COLOR CHARTS. 1971. Munsell Color Company, Inc., Baltimore, Maryland, U.S.A.

PEÑALOZA, R; GREZ, R, DONOSO, C. y CERDA, L. 1982. Bases técnicas de los tratamientos silvícolas para la regeneración y utilización de los bosques de la zona costera de Aysén. I. Reconocimiento sector Isla Magdalena. Informe de Convenio $\mathrm{N}^{\circ} 47$, Facultad de Ciencias Forestales, Universidad Austral de Chile, 37 pp. 